\title{
EXPERIENCIAS DE PROYECTO DE VINCULACIÓN CON LA COMUNIDAD MEDIANTE LA LÍNEA DE INVESTIGACIÓN COMERCIAL ADMINISTRATIVA ENFOCADA EN LA DIRECCIÓN ESTRATÉGICA DEL INSTITUTO SUPERIOR TECNOLÓGICO DE FORMACIÓN PROFESIONAL ADMINISTRATIVA Y COMERCIAL
}

\author{
Lissette Juleysi Vacacela \\ Conforme, Ing. \\ Instituto Superior Tecnológico de \\ Formación Profesional \\ Administrativa y \\ Comercial \\ lissette.vacacela@formacion.edu \\ .ec
}

\author{
Christopher Andrés Carchipulla \\ Alvarado, Ing. \\ Instituto Superior Tecnológico de \\ Formación Profesional \\ Administrativa y \\ Comercial \\ christopher.carchipulla@formacion. \\ edu.ec
}

\author{
Cesar Andrés Valenzuela Velasco, \\ Ing. \\ Instituto Superior Tecnológico de \\ Formación Profesional \\ Administrativa y \\ Comercial
}

cesar.valenzuela@formacion.edu.ec
Palabras claves: proyecto, vinculación, sociedad, capacitación.

Keywords: project, linkage, society, training.
Recibido: 30 de Octubre de 2017

Aceptado: 29 de Noviembre de 2017

\section{RESUMEN}

En la presente ponencia se muestra el desarrollo de acciones de vinculación con los sectores social y productivo, a través de la concertación de programas y proyectos que fortalecen el proceso de enseñanza-aprendizaje, esta estrategia educativa permite formar profesionales íntegros con una verdadera conciencia y responsabilidad social y ambiental, contribuyendo al análisis y solución de problemas específicos de la comunidad. Se podrá denotar el proceso para la ejecución de los proyectos de desarrollo social y la importancia del rol docente en las estrategias y metodologías que apuntan al cumplimiento de los resultados esperados.

\begin{abstract}
In this rapporteur present the development of actions shown linkage with social and productive sectors, through the coordination of programs and projects that strengthen the teaching-learning process, this educational strategy allows to form integral professionals with a true conscience and social and environmental responsibility, contributing in the analysis and solution of specific problems of the community. It may denote the process for the implementation of projects of social development and the importance of the teaching role in the strategies and methodologies aimed at meeting the expected results.
\end{abstract}




\section{INTRODUCCIÓN}

El estudiante de las instituciones de educación superior (IES) tiene como propósito fundamental aprender mediante la utilización de estrategias de aprendizaje productivas y desarrolladoras, cuya base estará siempre orientada al desarrollo de su conciencia social de acuerdo a las competencias generales y específicas de su carrera en correspondencia con el modelo del profesional. El desarrollo de las prácticas pre-profesionales y proyectos de vinculación con la comunidad son vitales para lograr los objetivos formativos de los tecnólogos en las (IES). De manera que la sociedad aporte constantemente a la academia, y a su vez, la academia también aporte a la sociedad mediante el desarrollo de nuevos proyectos guiados hacia el desarrollo sostenible y a la matriz productiva del país.

En la actualidad la visión y la misión de la universidad ecuatoriana no es solamente formar profesionales, sino hacerlo en el marco de las necesidades de la población, con sentido crítico y con identidad nacional. En este sentido, la vinculación con la sociedad es trascendental, esta se sustenta en cuatro pilares fundamentales: las prácticas pre profesionales, los trabajos comunitarios, la educación continua y un área de asesorías, servicios, movilidad y eventos. En el Reglamento de Régimen Académico para la universidad ecuatoriana del Consejo de Educación Superior, del 17 de diciembre de 2014, el Título VI, Vinculación con la Sociedad, en el Capítulo I, Artículo 77, Pertinencia de las carreras y programas académicos, se señala: "Se entenderá como pertinencia de carreras y programas académicos a la articulación de la oferta formativa, de investigación y de vinculación con la sociedad, con el régimen constitucional del Buen Vivir, el Plan Nacional de Desarrollo, los planes regionales y locales, los requerimientos sociales en cada nivel territorial y las corrientes internacionales científicas y humanísticas de pensamiento".

El Instituto Superior Tecnológico de Formación Profesional Administrativa y comercial (ITF), considera la Vinculación con la Sociedad como el proceso sustantivo orientado a generar un sistema integrador entre el instituto y su entorno, que apoye la investigación, gestión academia y la docencia, a través de la ejecución de programas y proyectos multidisciplinarios de desarrollo. Se cumple también de este modo con los artículos 87 y 88 de la Ley Orgánica de la Educación Superior donde se especifica que el estudiante deberá cumplir con servicios a la comunidad beneficiando a sectores rurales y marginados de la población. (CES, 2013).

Cabe mencionar que en el proceso de enseñanza y aprendizaje que se genera en el entorno áulico del (ITF) se valora cómo se puede aportar a la sociedad y también cómo mejorar la productividad de las de empresas utilizando diferentes herramientas, vías, estrategias y alternativas. Siendo una fortaleza el diseño de proyectos de vinculación en base a las competencias y conocimientos que poseen los estudiantes de la institución en temas relacionados con la Administración para promover y desarrollar el espíritu emprendedor, plasmando las ideas de negocios de los habitantes de las zonas rurales y vulnerables del país, con la máxima que una idea innovadora puede producir un cambio social significativo. 
El objetivo principal del presente artículo es socializar los resultados y las experiencias adquiridas a partir de la implementación del proyecto de vinculación ejecutado en la provincia de Santa Elena por los estudiantes de Administración de Empresas del ITF, donde se expone el proceso que se llevó a efecto para garantizar la efectividad del mismo.

\section{DESARROLLO}

La academia fue siempre base de la construcción de sociedades y para la trasmisión de los mejores conocimientos acumulados por la humanidad, sin embargo, aún se mantiene una deuda histórica frente al país, relacionada con lo que puede aportar la academia para hacer una sociedad más próspera, sustentable y desarrollada, en tal dirección aún hay mucho por hacer en pro de la comunidad.

El gobierno del Ecuador, específicamente el Ministerio de Educación, desarrolla desde hace ya muchos años el proyecto de Vinculación con la Sociedad, el cual no solo ayuda a la sociedad, sino que contribuye a que el estudiante se interrelacione con la realidad del país, poniendo en práctica los conocimientos adquiridos e interactuando con ella desde sus habilidades y competencias profesionales a fin de lograr un perfil profesional mucho más realista. Por tal razón, el Instituto Superior Tecnológico de Formación Profesional Administrativa y Comercial (ITF) por medio del departamento de Vinculación con la Sociedad, se proyecta socialmente para intervenir en la solución de los problemas de la comunidad, aportando en el ámbito social, ecológico y económico.

Se considera que uno de propósitos de los proyectos de vinculación debe estar enfocado a la capacitación permanente de la sociedad, teniendo en cuenta, entre otras razones, que muchos comerciantes o empresas familiares fracasan por falta de capacitación, siendo del criterio que a través del aprendizaje y la capacitación se aportan a las personas los conocimientos adecuados y las preparan para el desarrollo de sus funciones en una determinada labor profesional.

Es una forma de dar respuestas a los objetivos 3 y 4 del Plan Nacional del Buen Vivir que buscan mejorar la calidad de vida de la población y fortalecer las capacidades y potencialidades de la ciudadanía.

La educación y el aprendizaje han sido dos procesos que desde la época primitiva han posibilitado el intercambio de conocimientos y habilidades en pos del crecimiento personal y profesional de los individuos para alcanzar sus objetivos.

El poder del entrenamiento y la perseverancia para adquirir conocimientos, desarrollar habilidades y competencias profesionales es sin lugar a dudas un elemento movilizador del hacer y actuar de los seres humanos. Poco a poco los procesos de estudio y entrenamiento se han ido sistematizando en todos los campos de la ciencia mejorando las capacidades de las personas. 
La Real Academia de la Lengua Española define capacitar como hacer a alguien apto, habilitarlo para algo. Otra definición de Gallardo (2014), menciona a la capacitación como un conjunto de medios que se organizan de acuerdo a un plan, para lograr que un individuo o grupo adquiera destrezas, valores o conocimientos teóricos, que le permitan realizar ciertas tareas o desempeñarse en algún ámbito específico, con mayor eficacia.

En los proyectos que se han desarrollado para fomentar el vínculo con la sociedad se han podido apreciar diferentes problemáticas y situaciones, entre las cuales se puede mencionar la desmotivación con que empiezan los estudiantes para incorporarse a proyectos de vinculación, lo cual se acrecienta por el rechazo que muestran algunas comunidades al escucharlos, debido a que conviven en ellas muchos comerciantes empíricamente formados los cuales no ven la necesidad de formar equipos de trabajo organizados por estudiantes, mucho menos de trabajar en base a objetivos, de desarrollar el talento humano, de mejorar el servicio al cliente, inclusive de ser un profesional ético.

Atendiendo a lo expresado anteriormente se hace necesario acometer una fase inicial de sensibilización, motivación y concientización en los estudiantes de la necesidad de crear y llegar a ser un mejor profesional, comprometido con su profesión, con la sociedad y consigo mismo. Consecuentemente, deben evaluarse las mejores opciones para poder desarrollar los proyectos de vinculación durante la carrera.

Para ello hay que tener en cuenta las condiciones, los recursos, los horarios de trabajo, el lugar, etc., teniendo como premisa la caracterización de los estudiantes, ya que tienen diferentes potencialidades, poseen diferentes niveles, ritmos y estrategias de aprendizaje, diferentes motivaciones, aspiraciones, necesidades, carencias, viven en diferentes provincias, entre otras peculiaridades. Se pueden encontrar otros inconvenientes para lograr los objetivos de los proyectos de vinculación, como, por ejemplo: la falta de trabajo de equipo entre los estudiantes, por lo que se hace imprescindible motivarlos a formar grupos de trabajo, convenir horarios, acordar objetivos individuales coherentes con. los objetivos grupales en pro de la sociedad.

El rol del docente tutor debe estar enfocado hacia los objetivos del proyecto, el trabajo en equipo, el proceso investigativo, las técnicas que se utilizan para poder llegar a los vinculados, la elaboración del material de apoyo que necesita el estudiante, la orientación de las nuevas tecnologías, la creación de las condiciones: transporte, recursos y medios profesionales. Especial atención ha de darse a la parte humana del estudiante, para promover en ellos su motivación, disposición, rendimiento e implicación en las acciones y actividades del proyecto.

La singularidad y alcance de la creación de los proyectos de vinculación está en que permite la relación del contenido teórico - práctico de acuerdo a las necesidades existentes en la comunidad, asociación, gremio, etc., para facilitar el aprendizaje y generar una comprensión más efectiva. De manera, que a largo plazo se pueda generar un impacto social que beneficie a más personas. 
Para la implementación de un proyecto se incluyen cuatro etapas que permiten tener una planificación donde se pueda medir los resultados. Estas son: el diagnóstico, diseño, monitoreo y evaluación de los proyectos de desarrollo social cuyo alcance involucra al departamento de Vinculación con la Sociedad, comisión de vinculación, a todos los directores de carrera y responsables de proyecto.

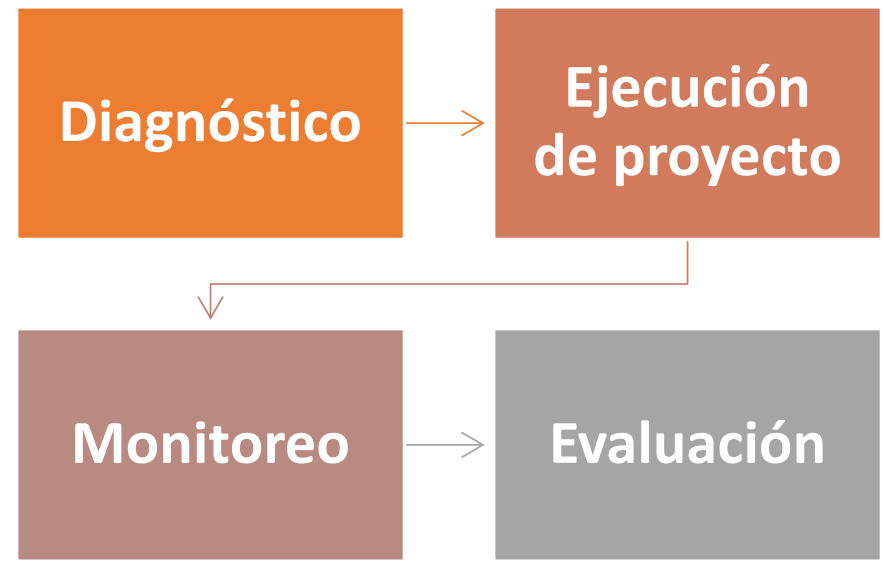

En la etapa de diagnóstico se evidencia la situación actual en el contexto social, económico, ambiental y cultural; se identifica el porqué del proyecto y la problemática existente por medio de recolección de datos que pueden ser cualitativos y cuantitativos.

Posteriormente se define el diseño del proyecto sobre la base de la información proporcionada en el diagnóstico; una vez el mismo esté planteado se realiza la ejecución; para el seguimiento se realiza el monitoreo a partir de indicadores precisos, se parte de una línea base que permita tener una realidad establecida frente a una esperada a partir de la intervención. Finalmente se evalúa el progreso de los resultados.

En correspondencia, y siguiendo como basamento la fundamentación brindada con anterioridad, se procedió para diseñar, planificar, organizar, ejecutar y evaluar el proyecto de vinculación en la carrera de Administración de Empresa del ITF.

Se escogió como lugar de trabajo "La Libertad" debido a la cercana relación con el municipio de dicha ciudad. La cooperativa escogida se llama Rafael Correa Delgado, se caracterizó por abrir las puertas a los estudiantes, asumiendo sus objetivos con motivación y deseos de cooperar con ellos. 
El sector es un lugar suburbano, de difícil acceso, con calles de tierra sin pavimentar, casas en obra muerta o algunas otras de caña, pero con una calidez de su gente muy notable. Ellos se encuentran organizados con una junta directiva, el presidente tiene la máxima responsabilidad por el cumplimiento de los objetivos, el éxito de su organización y está a cargo de organizar reuniones de interés de la comunidad.

En sinergia con todo esbozado, los estudiantes de la mencionada carrera estructuraron en armonía con los docentes y tutores el proyecto de vinculación, que incluyó un plan de capacitación en técnicas administrativas para los pequeños empresarios de la comunidad, donde intervinieron 9 mujeres y 7 hombres, formando un $56.2 \%$ mujeres y $43.7 \%$ de hombres.

Un dato interesante es la motivación, sensibilización y concientización lograda en el 62.5\% de los estudiantes, los cuales no son de la ciudad de Guayaquil, sino de diversas provincias de La Costa, 2 de ellos tienen que viajar 4 horas y media para llegar al destino y el 100\% trabajan, motivo por el cual estudian en horarios de fines de semana dejando el domingo para el desarrollo del proyecto. El 100\% de los estudiantes mantienen un rango de edad entre 20 y 25 años, actualmente se encuentran cruzando el quinto nivel de la carrera.

Inicialmente se realizó un levantamiento de información mediante el desarrollo de entrevistas y encuestas a los participantes que decidieron intervenir en el proyecto. Entre las principales respuestas que se valoraron están: el $80 \%$ de los encuestados son vendedores informales, es decir trabajan en la calle a diario, comprando mercadería de temporada para poder producir ya que no cuentan con instrucción superior, y en muchos casos, no han terminado el colegio, por lo que no poseen puestos de trabajo en las empresas ni públicas ni privadas.

Más del $75 \%$ son inmigrantes de otras provincias que decidieron empezar nuevamente en otra ciudad del país dejando atrás un pasado sin éxito, con muchas carencias materiales y muchos de ellos afligidos por serios problemas, familiares y sociales, haciéndose responsables de familias a muy temprana edad.

De acuerdo a las necesidades de los actores involucrados, el proyecto de vinculación se centró en el área comercial, articulada a la línea de investigación Comercial Administrativa en relación a sus ejes de gestión empresarial y dirección estratégica. Abarca entre otros contenidos: la responsabilidad social de la empresa, trabajo en equipo y liderazgo, cultura de la calidad de la gestión y desarrollo de planes de negocios.

Se direcciona la materia Comportamiento Profesional y Ambiental, en la cual se desarrollan competencias enfocadas hacia el desarrollo sostenible, fortaleciendo los tres (3) pilares fundamentales del proyecto: la sociedad, el medio ambiente y el desarrollo económico. Otra materia necesaria para el desarrollo del proyecto fue Gerencia de Servicios en la cual se profundiza en el estudio de una cultura enfocada hacia el cliente, basada en la mejora de los procesos para lograr una óptima calidad de los productos o servicios. 
La plataforma del proyecto se direccionó en lo fundamental en el manejo apropiado de ingresos, ya que muchas veces gastar dinero en cosas innecesarias o llenarse de deudas es algo que los caracteriza. Son personas de escasos recursos, chulqueros con altos intereses, atados a vivir con más gastos de lo que se ingresa. Consiguientemente, se diseñó un formato para el control de ingresos y egresos, al final del mes cada persona puede evaluar los rubros de gastos innecesarios que se hace para poder organizar mejor sus finanzas personales.

Una de experiencias que puede mostrase como resultado positivo del proyecto es el cambio paulatino que se fue observando en los participantes, sobre todo en el momento de comprobar financieramente lo que gastan, comenzaron poco a poco a valorar más el dinero, se pudo observar en ellos como se fueron motivando por ahorrar más, a no pagar intereses elevados.

Otro resultado importante estuvo basado en la vinculación de las personas con el ejercicio práctico de sus ventas, lo que contribuyó al mejoramiento del servicio al cliente, del proceso y su producto, minimizando costos para obtener un mejor margen de utilidad neta sin minimizar la calidad del producto. Por supuesto, esto fue despertando el interés por convertirse en un profesional ético, respetuoso del cliente y contribuidor al desarrollo para el país. No obstante, la esencialidad de este proyecto fue elevar la capacitación del sector anteriormente mencionado lográndose plenamente los objetivos propuestos.

También se capacitaron 20 microempresarios, los cuales carecían de herramientas administrativas que les permitieran alcanzar los objetivos planteados, en la dinámica de este proceso se realizó una evaluación donde se valoró específicamente los siguientes aspectos:

\begin{tabular}{|c|c|c|c|c|}
\hline Capacitación & Excelente & Muy bueno & Bueno & Total \\
\hline $\begin{array}{c}\text { Pertenecía de los } \\
\text { contenidos } \\
\text { impartidos }\end{array}$ & 16 & 4 & & 20 \\
\hline $\begin{array}{c}\text { Actualidad de los } \\
\text { contenidos } \\
\text { socializados }\end{array}$ & 18 & 2 & & 20 \\
\hline $\begin{array}{c}\text { Relación de los } \\
\text { contenidos } \\
\text { impartidos con su } \\
\text { entorno laboral }\end{array}$ & 20 & & & \\
\hline
\end{tabular}




\section{Pertinencia de la capacitación}

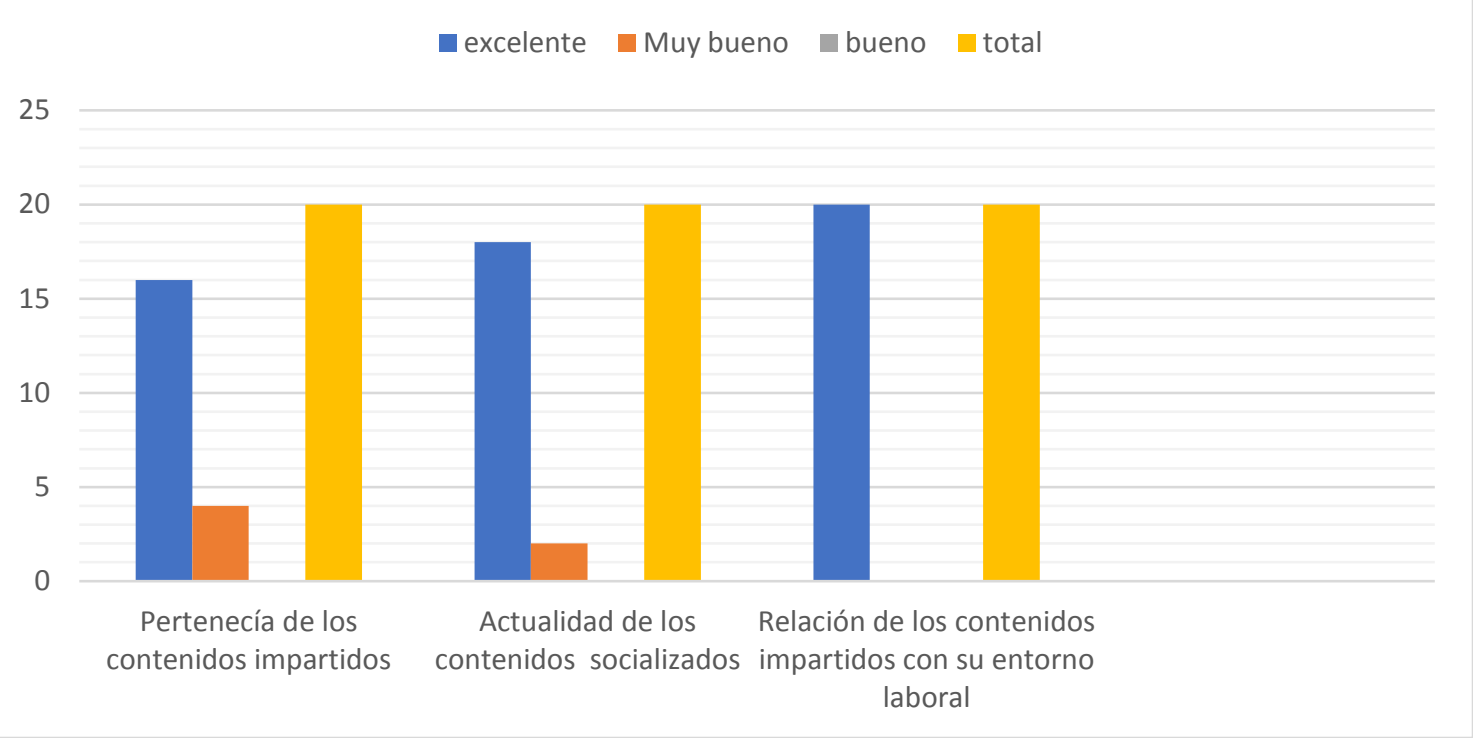

\section{Elaborado por el autor}

Como se puede valorar en la gráfica la capacitación impartida por los estudiantes del ITF, a través de las acciones del proyecto de vinculación, tuvo un alto impacto en la sociedad y especialmente en la cooperativa Rafael Correa Delgado.

De manera muy general entre los resultados alcanzados por el proyecto se pueden mencionar:

- En los estudiantes de la carrera de Administración de empresas del ITF se logró elevar tangiblemente: la motivación por la realización de proyectos de vinculación, el desarrollo de habilidades profesionales, el vínculo de la teoría con la práctica profesional, mayor conciencia de su papel en el desarrollo de sociedad, en particular de las comunidades más desfavorecidas y de su gente más carente de conocimientos para poder sobrevivir y ayudar a su familia.

- En el caso de los participantes de la comunidad en el proyecto se fue manifestando gradualmente:

- La adquisición de conocimientos útiles para el desarrollo de las ventas de los productos ofrecidos.

- La implementación y el uso de las herramientas tecnológicas para la implementación de sus microempresas.

- El fortalecimiento de las capacidades de negociación y planificación de sus finanzas personales. 
- La generación de nuevas ideas de inversión mediante un reconocimiento del mercado objetivo, ayudando no solamente a sus familias sino al mejoramiento de economía zonal y su inserción a la economía cantonal.

Debido a la incidencia positiva que alcanzó este proyecto se continuará, a petición de la comunidad, aplicando en una segunda etapa en el período de febrero 2018 a julio de 2018, donde se capacitará a los comunitarios interesados con nuevos requerimientos desde otras perspectivas de comercio, venta, negocios, administración y otras, solicitados por la comunidad por su gran repercusión en lo personal, familiar y social.

La experiencia mostrada constituye un intento valioso que demuestra la importancia y el cómo puede lograrse a través de la investigación acción la realización de proyectos de vinculación con la sociedad, así como la manera en que este proceso sustantivo puede insertarse dentro de las líneas de investigación comercial administrativa en el ITF, particularmente aquella enfocada en la dirección estratégica de la institución, constituye una manera de visualizar como lo que se está proyectando y haciendo va teniendo una alta incidencia en el proceso formativo del profesional.

\section{CONCLUSIÓN}

Se ha evidenciado que a través de los proyectos de vinculación se logra enriquecer los conocimientos de los estudiantes con vivencias reales, convirtiéndose en agentes generadores de cambios significativos e innovadores que aportan a la sociedad.

El tecnólogo que cursa la carrera de Administración de empresas en el ITF contribuyó desde su accionar durante el proyecto de vinculación a impulsar las ideas de negocios de las comunidades, al mismo tiempo se favoreció la adquisición de nuevas experiencias profesionales y conocimientos científicos y prácticos vinculados a la capacitación de emprendedores.

Las capacitaciones realizadas, como parte del proyecto de vinculación, permitieron el desarrollo de conocimientos y habilidades específicas para el mejoramiento de los comerciantes de la provincia de Santa Elena en la cooperativa Rafael Correa Delgado, dotándolos de conocimientos valiosos y pertinentes para poder realizar mejor su labor.

Se elevaron los niveles de satisfacción de los estudiantes integrantes del proyecto por compartir con la comunidad los conocimientos adquiridos durante su carrera, sus experiencias profesionales, la aplicación de habilidades prácticas, su cultura profesional y valores profesionales y humanos, siendo una forma de cumplir con la misión del instituto de formar profesionales y ciudadanos con responsabilidad social y ambiental, capaces de diseñar, ejecutar y evaluar procesos relacionados con la actividad productiva e innovación tecnológica. 


\section{BIBLIOGRAFÍA}

1. Alcántar, V. M. y Arcos, J. L. (2004). La vinculación como instrumento de imagen y posicionamiento de las instituciones de educación superior. Revista Electrónica de Investigación Educativa.

2. CES. (2014). Reglamento de Régimen Académico para la universidad ecuatoriana del Consejo de Educación Superior del 17 de diciembre de 2014. Ecuador.

3. CES. (2013). SNNA. Recuperado de http://www.snna.gob.ec/wpcontent/themes/institucion/dw-pages/Descargas/regimen_academico.pdf

4. Fuentes H. (2000). La Universidad y sus procesos. Centro de Estudios de Educación Superior. Universidad de Oriente. Cuba.

5. Hermida B, C. (2017). La universidad y su vinculación con la sociedad. Recuperado de http://www.eltiempo.com.ec/noticias/columnistas/1/394821/la-universidad-y-suvinculacion-con-la-sociedad

6. LOES. (2010). Consejo Nacional para la Igualdad Intergeneracional. Recuperado de Ley

7. Orgánica de Educación Superior: http://www.igualdad.gob.ec/docman/generales/1965-ley-organica-educacionsuperior-loes/file.html

8. Malagón, L. A. (2006). La vinculación universidad-sociedad desde una perspectiva social. Educación y Educadores 